
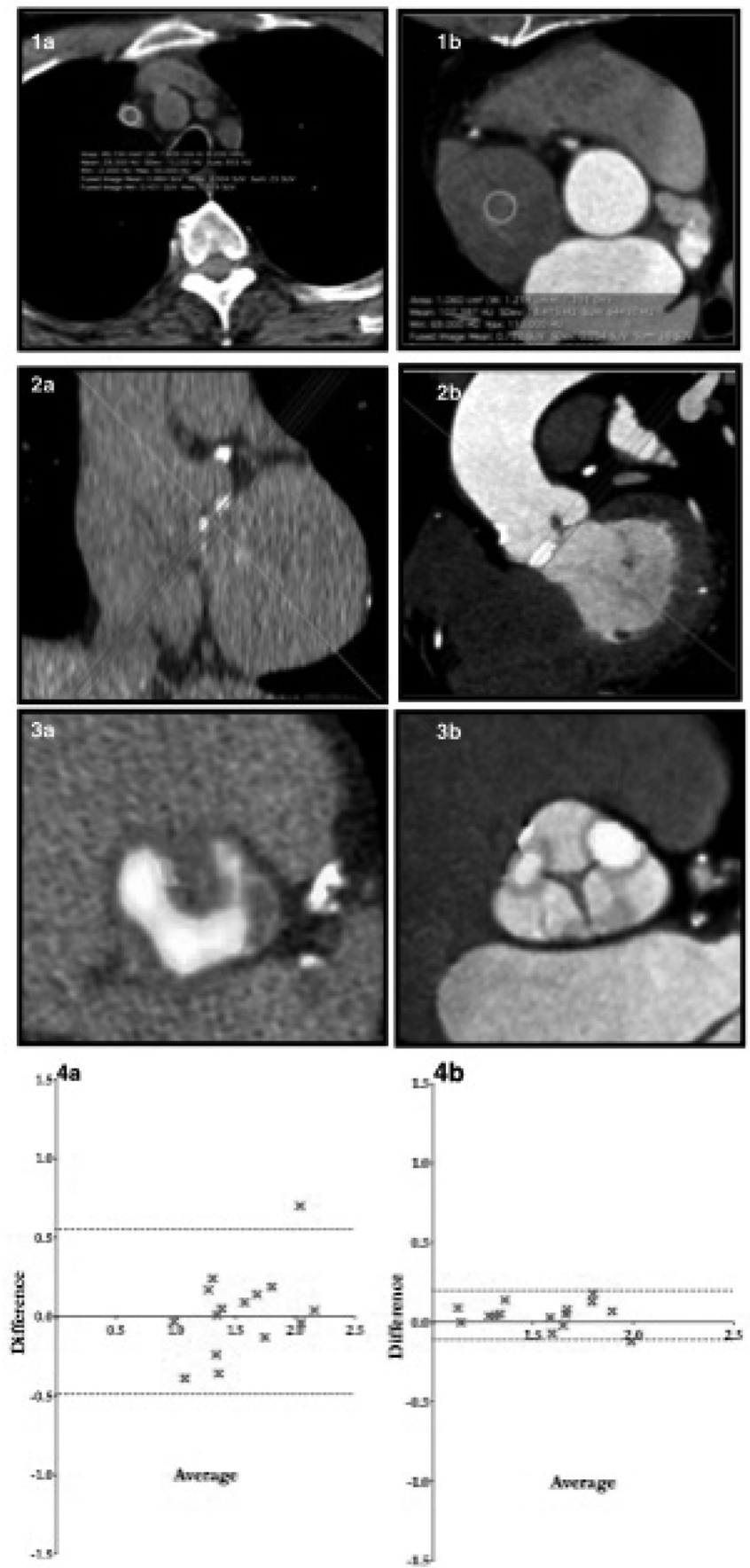

Abstract 117 Figure 1 Blood pool correction was performed by sampling from the right atrium instedad of the brachio-cephalic vein. 2 . The most diseased segment method was used to quantify the PET signal within 2 hottest slices rather than the whole valve/ 3 . Contrase CT fused with ECG-gated PET gacilitated better localization to leaflets compared to the original approach 4. Bland-Altmanplots emonstrated superior sacn-rescan reproducibility for the modified technique (2b) compared to the previous approach (2a)

may also be possible to subtract materials from each other, such as calcium from iodine.

Single source DECT, using two energy spectra from a single anode almost simultaneously using rapid tube voltage switching, requires novel detector technology capable of distinguishing signals $0.25 \mathrm{~ms}$ apart without artefact from detector artefact. To date, this has not been evaluated in patients with coronary calcification. This feasibility study examined potential benefits and limitations of virtual monochromatic and material decomposition images for assessing calcified coronary arteries, and their potential diagnostic accuracy compared to invasive angiography.

Methods The study was approved by the National Research Ethics Service. Patients gave informed, written consent. Thirty patients undergoing invasive angiography on clinical grounds, with evidence of coronary calcification, underwent CTCA with a single-source DECT scanner. The results of each test were assessed by experienced, independent, blinded readers and compared in per-segment, per-vessel and per-patient analyses.

Results 403 segments in 86 vessels were analysed. The median Agatston score was 964. The accuracy of virtual monochromatic imaging is outlined in Table 1 . Overall the accuracy for the identification of moderate and severe stenosis was 0.88 and 0.88 on a per-segment basis, 0.84 and 0.86 per vessel, and 0.93 and 0.97 per-patient. The weighted kappa score between invasive and CT angiography was 0.71 suggesting good agreement.

The per-segment sensitivity, specificity, PPV and NPV (and $95 \%$ confidence intervals) of the material decomposition images were $0.67(0.57-0.76), 0.82(0.77-0.86), 0.54$ (0.45$0.63)$ and $0.88(0.84-0.92)$ respectively for moderate stenosis, and $0.70(0.57-0.80), 0.79(0.75-0.83), 0.40(0.31-0.49)$ and $0.93(0.89-0.96)$ respectively for severe stenosis. Overall accuracy was 0.78 for both moderate and severe stenosis. Calcium subtraction was highly inconsistent, mainly due to image noise with resultant misidentification of calcium and excessive subtraction.

Conclusions The study suggests that single source DECT is feasible in patients with severe coronary calcification, and virtual monochromatic imaging may improve accuracy compared to conventional CT. A larger study comparing standard and DECT is merited.

\section{THE EFFECT OF PRELOAD REDUCTION USING HEAD-UP TILT TESTING: AN EXPLORATORY STUDY USING LEFT VENTRICULAR LONGITUDINAL AND TRANSVERSE STRAIN-VOLUME LOOPS}

${ }^{1}$ David Oxborough, ${ }^{1}$ Tom Bracknall, ${ }^{1}$ Rachel Lord, 'Lynsey Forsythe*, ${ }^{1}$ Mohammad Qasem, ${ }^{1}$ Punit Mistry, ${ }^{2}$ John Somauroo, ${ }^{1}$ Keith George. 'Liverpool John Moores University; ${ }^{2}$ Countess of Chester Foundation Hospital NHS Trust; *Presenting Author

\subsection{6/heartjnl-2016-309890.119}

Introduction Left ventricular (LV) function is dependent on intrinsic contractility and relaxation as well as the prevailing loading conditions. The Frank-Starling mechanism states that

\section{Abstract 118 Table 1}

\begin{tabular}{lllll}
\hline & & Per segment & Per vessel & Per patient \\
\hline$>50 \%$ stenosis & Sensitivity & $0.76(0.66-0.84)$ & $0.78(0.64-0.89)$ & $0.93(0.76-0.99)$ \\
& Specificity & $0.92(0.89-0.95)$ & $0.93(0.80-0.98)$ & $1.00(0.19-1.00)$ \\
& PPV & $0.76(0.66-0.84)$ & $0.92(0.79-0.98)$ & $1.00(0.86-1.00)$ \\
& NPV & $0.92(0.89-0.95)$ & $0.79(0.65-0.90)$ & $0.50(0.08-0.92)$ \\
& & & & \\
& & & & \\
& Sensitivity & $0.73(0.60-0.83)$ & $0.78(0.61-0.90)$ & $1.00(0.85-1.00)$ \\
& Specificity & $0.91(0.87-0.93)$ & $0.94(0.84-0.98)$ & $0.83(0.36-0.97)$ \\
& PPV & $0.58(0.47-0.70)$ & $0.90(0.74-0.98)$ & $0.96(0.79-0.99)$ \\
& NPV & $0.95(0.92-0.97)$ & $0.86(0.74-0.94)$ & $1.00(0.48-1.00)$ \\
\hline
\end{tabular}


absolute myocardial shortening would be lower in the presence of a reduced preload, however LV function is complex and must adapt in this setting in order to maintain stroke volume and cardiac output. The impact of preload reduction on cardiac mechanics has been scarcely explored however the direct relationship of strain (s) in different planes and simultaneous volume assessment has not been assessed. This exploratory study utilises a novel technique (s-volume loops) in order to establish the relative temporal contribution of longitudinal (Ls) and transverse strain (Ts) to volume change throughout the cardiac cycle following 1 and $5 \mathrm{~min}$ of head-up tilt testing.

Methods Five healthy subjects underwent standard transthoracic echocardiography to obtain an apical 4 chamber orientation with a focus on the $\mathrm{LV}$ in a supine position and following $1 \mathrm{~min}$ and $5 \mathrm{~min}$ of head-up tilt testing. As well as a standard assessment, raw temporal Ls and Ts values were exported and divided into 5\% time increments across the cardiac cycle. Concomitant LV volumes were traced at each 5\% time increment to provide simultaneous s-volume loops. The s-volume relationship was assessed by 1) polynomial equations to derive absolute strain values for $\%$ end diastolic volumes (EDV) and 2) systolicï $\dot{1}^{1} / 2$ diastolic coupling (SDcoup) as the difference between systolic and diastolic strain within the same working range of\% EDV.

Results There was a significant reduction in EDV at 1 min of headup tilting $\left(92 \ddot{i} \dot{c}^{1} / 216 \mathrm{ml}\right.$ Vs. $\left.64 \ddot{i}^{1} 1 / 26 \mathrm{ml}\right)$ with no further change at 5 min. There was no significant change in EF or effective stroke volume. There was a reduction in peak Ls at 1 min tilting $\left(-16 \ddot{\mathrm{i}}^{1} 1 / 21\right.$ Vs. $\left.-12 \ddot{i} \dot{c}^{1 / 2} 1 \%\right)$ which remained stable at $5 \mathrm{~min}$. Conversely, there was a gradual increase in Ts across time (16 i $\mathrm{c}^{1} / 23$ Vs. $37 \mathrm{i}^{1} \mathrm{1} / 211 \%$ ) as well as a gradual reduction in the time to peak Ts. Following calculation of s-volume loops the paradoxical relationship of reduced Ls and a concomitant increase in Ts remained at all 10\% increments of EDV. There was a marked increase in Ts SDcoup at all 10\% EDV increments at 5 min post tilt.

Conclusion Preload reduction as a consequence of head-up tilt testing results in a shift in cardiac mechanics with a reduction in longitudinal and concomitant increase in transverse contribution to volume reduction and maintenance of EF. The systolic-diastolic coupling suggests that LV filling in the setting of a reduced preload is maintained by a greater return to original length of the myocardium in the transverse plane for any given volume. These findings provide important physiological value when assessing LV function in the clinical setting where a reduction in preload is evident.

Abstract 119 Table 1 Conventional echocardiographic indices at supine, 1 minute and 5 minutes of head-up tilting

\begin{tabular}{|c|c|c|c|}
\hline Conventional Parameters & SUPINE & 1 MIN TILT & 5 MIN TILT \\
\hline Heart Rate (bpm) & $77 i_{i}{ }^{1} / 26$ & $90 \ddot{i} i^{1 / 2} 9^{*}$ & $87 \ddot{i} i^{1 / 2} 6^{\wedge}$ \\
\hline Systolic Blood Pressure (mmHg) & $127 i i^{1 / 2} 8$ & $125 i_{i}^{i} / 210$ & $127 i_{i} i^{1 / 2} 7$ \\
\hline Diastolic Blood Pressure (mmHg) & $76 i_{i}^{1} 1 / 27$ & $79 \ddot{i} i^{1 / 2} 15$ & $83 i i^{1 / 2} 5$ \\
\hline Cardiac Output (I/min) & $4.3 i_{i}^{1} / 20.6$ & $3.9 i_{i}^{1} / 20.2$ & $3.8 i_{i}^{1} / 20.7$ \\
\hline End Diastolic Volume (ml) & $92 \ddot{i}^{1} 1 / 216$ & $64 \ddot{i}_{i}^{1 / 2} 6^{*}$ & $69 \ddot{i} \iota^{1 / 211 \wedge}$ \\
\hline End Systolic Volume (ml) & $37 i i^{1} / 27$ & $20 \ddot{i} i^{1 / 2} 6$ * & $25 \ddot{i} i^{1 / 2} 7$ \\
\hline LV Ejection Fraction (\%) & $60 i i^{1 / 2} 4$ & $69 \ddot{i} i^{1} / 27$ & $63 i i^{1 / 2} 6$ \\
\hline E Wave Velocity (m/s) & $0.81 i_{i} i^{1} / 20.19$ & $0.78 \ddot{i}_{i}^{1} 1 / 20.09$ & $0.70 i^{1}{ }^{1 / 2} 0.10$ \\
\hline A Wave Velocity (m/s) & $0.55 \ddot{i}^{1}{ }^{1 / 2} 0.11$ & $0.59 i_{i} i^{1 / 2} 0.08$ & $0.66 i^{1}{ }^{1 / 2} 0.26$ \\
\hline$E / A$ & $1.5 i_{i}^{1 / 2} 0.6$ & $1.3 \ddot{i}_{i}^{1 / 2} 0.2$ & $1.2 \mathrm{i}_{\mathrm{i}}^{1} / 20.6$ \\
\hline
\end{tabular}

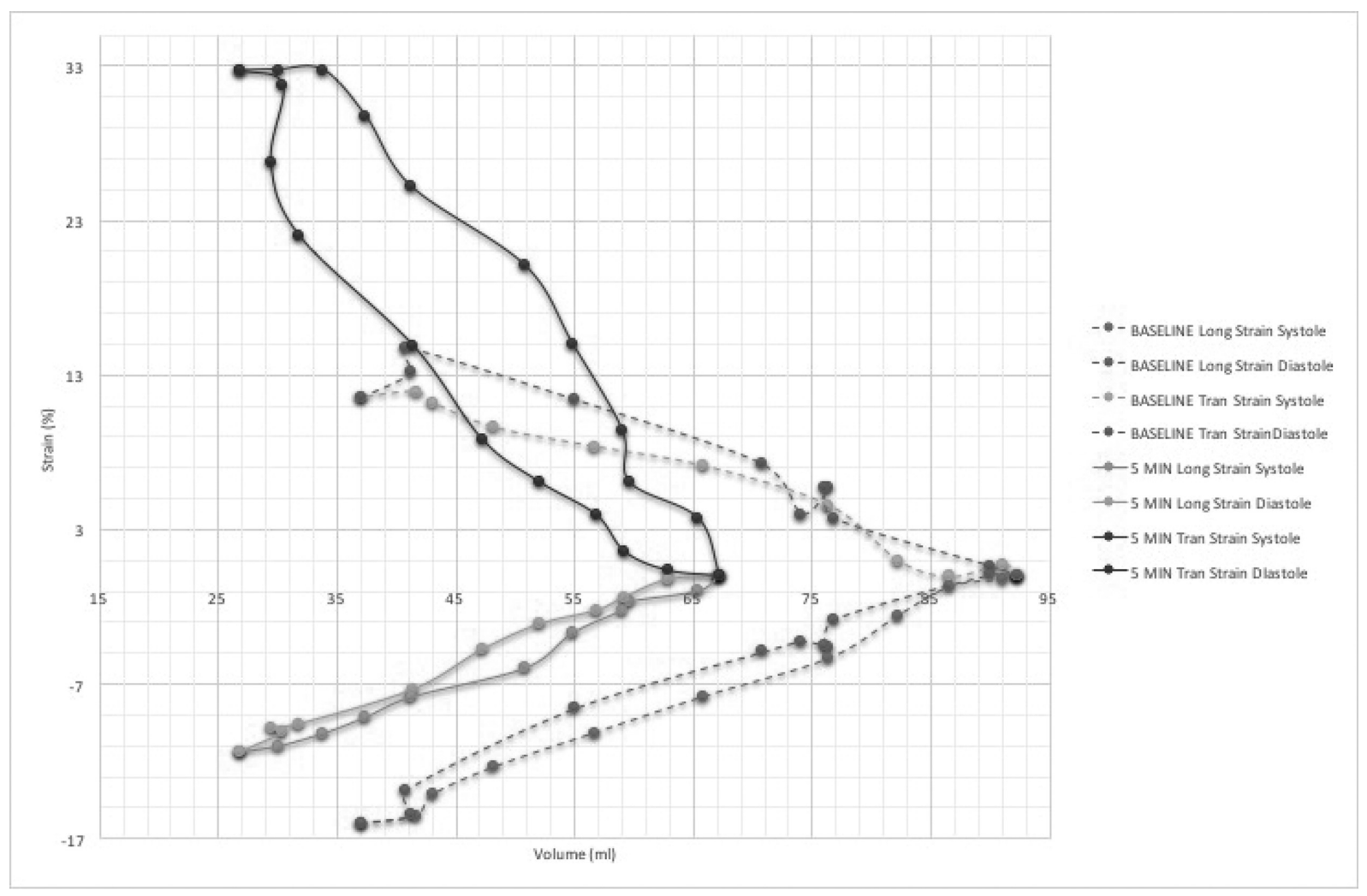

Abstract 119 Figure 1 Longitudinal and transverse strain-volume loops at supine and at 5 minutes head-up tilting 